\title{
Y-box Protein-1 Regulates the Expression of Collagen I in Hepatic Progenitor Cells via PDGFR- $\beta /$ ERK/p90RSK Signalling
}

\author{
Fei Li, ${ }^{1}$ Zhenzeng Ma, ${ }^{1}$ Heng Liu, ${ }^{2}$ Qidi Zhang, ${ }^{1}$ Xiaobo Cai, ${ }^{1}$ Ying Qu, ${ }^{1}$ Mingyi Xu, ${ }^{1}$ and \\ Lungen $\mathrm{Lu}^{1}$ \\ ${ }^{1}$ Department of Gastroenterology and Hepatology, Shanghai General Hospital, Shanghai Jiao Tong University School of Medicine, \\ Shanghai 200080, China \\ ${ }^{2}$ Gastroenterology Department, The First Affiliated Hospital of Anhui Medical University, Hefei, Anhui 230022, China
}

Correspondence should be addressed to Mingyi Xu; xumingyi2014@163.com and Lungen Lu; lungenlu1965@163.com

Received 14 January 2017; Accepted 6 April 2017; Published 27 August 2017

Academic Editor: Luca Vanella

Copyright ( 2017 Fei Li et al. This is an open access article distributed under the Creative Commons Attribution License, which permits unrestricted use, distribution, and reproduction in any medium, provided the original work is properly cited.

\begin{abstract}
Y-box protein-1 (YB-1) is a highly conserved transcription factor that is involved in multiple biological processes via transcriptional regulation of several genes, including $p 53$, cyclin D1, and EGFR. YB-1 has been reported to be overexpressed in injured livers. This study aims to explore the functions of YB-1 in hepatic progenitor cells (HPCs). Herein, chromatin immunoprecipitation sequencing (ChIP-sequencing) and RNA-sequencing assays identified that YB-1 participated in the biological adhesion process and ECM-receptor interactions in HPCs. Further study demonstrated that YB-1 modulated the expression of extracellular matrix components in HPCs. ChIP-sequencing assays established that PDGFR- $\beta$ was a target gene of YB-1, and luciferase reporter assays confirmed that YB-1 negatively regulated PDGFR- $\beta$ promoter activity in HPCs. In addition, PDGFR- $\beta$ can regulate the expression of collagen I through ERK/p90RSK signalling, and disruption of the signalling pathway with a PDGFR- $\beta$ inhibitor or ERK1/2 inhibitor abolished the regulatory effect of PDGFR- $\beta$ on collagen I expression in HPCs. Conclusively, YB-1 can modulate the expression of collagen I in HPCs via direct binding to the PDGFR- $\beta$ promoter, negatively regulating its expression. In addition, the ERK/p90RSK axis serves as the downstream signalling pathway of PDGFR- $\beta$.
\end{abstract}

\section{Introduction}

YB-1 belongs to a family of DNA- and RNA-binding factors, also named cold shock proteins, which are highly conserved during evolution and have been shown to function as regulators of gene transcription and translation. A wide range of nucleic acid structures are reported to be specifically bound to YB-1, most of which harbour an inverted CCAAT-box (ATTGG) as the core binding site. YB-1 was first recognized as a protein that binds to the promoter of the major histocompatibility complex II gene HLA-DR $\alpha$ and that negatively regulates the expression of this gene [1]. Later, a large number of studies regarding the functions of YB-1 were conducted, and they demonstrated multiple effects of YB-1 on cell proliferation, migration, and transformation. YB-1 directly interacts with $p 53$, and YB-1 knockdown upregulates endogenous $p 53$ and induces various tumour cell lines to undergo apoptosis $[2,3]$. Meanwhile, YB-1 has been found to bind to a number of genes, including cyclin D1, epithermal growth factor receptor (EGFR), and mitogen-activated protein kinase-interacting kinase 1 ( $M N K 1)$, and then modulate the transcription of these genes and the proliferation rate of related tumour cells [4-6]. Furthermore, YB-1 also participates in the production of the extracellular matrix (ECM) and in the scarring process. In human embryonic kidney cells and dermal fibroblasts, YB-1 exerted a repressive effect on the collagen $\alpha 1$ (I) (COL1A1) and matrix metalloproteinase-2 (MMP-2) gene promoters [7, 8].

YB-1 has been reported to be involved in liver development and liver diseases. Grant and his colleagues found that YB-1 was relatively abundant in the liver at day 7 of embryogenesis and decreased steadily throughout chicken 
TABle 1: Primer sequences for ChIP-PCR analysis.

\begin{tabular}{lllc}
\hline Primer & \multicolumn{1}{c}{ Forward sequence } & Reverse sequence & Product \\
\hline PDGFR- $\beta$ & CCAACTCAGGTGGCCTCTTA & TCACTGAACATCTGCCCTGT & $82 \mathrm{bp}$ \\
GAPDH & TGAGCTAGGACTGGTAAG & GTCCGTATTTATAGGAACCC & $166 \mathrm{bp}$ \\
\hline
\end{tabular}

embryogenesis. In addition, they found that YB-1 mRNA in rat livers was elevated approximately 10- and 6-fold 24 and $48 \mathrm{~h}$ after administration of carbon tetrachloride, respectively, which was accompanied by DNA synthesis and cell proliferation [9]. Gunasekaran et al. reported that most hepatocellular carcinoma tissues expressed YB-1 and showed a relatively higher expression compared to normal livers [10]. In addition, in vitro experiments demonstrated that YB-1 was a potent inducer of Smad7 expression in activated hepatic stellate cells (HSCs) and myofibroblastic mesangial cells, which could be used to antagonize TGF$\beta$ in chronic stages of fibroproliferative diseases in the liver [11]. In an inflamed liver, YB-1 suppressed the synthesis of collagen and modulated fibrogenesis [12].

Based on the above studies, we speculate that YB-1 may play certain roles in liver regeneration and fibrogenesis. In the present study, we attempted to determine the effects of YB-1 on hepatic progenitor cells, as well as the possible molecular mechanism. The results would help booster our understanding of liver repair and fibrogenesis.

\section{Materials and Methods}

2.1. Animals. C57BL/6J mice (4 weeks old) were purchased from the Sino-British Sippr/BK Laboratory and housed in the Animal Experimental Center of Shanghai First People's Hospital (Shanghai, China) under specific pathogen-free conditions. The Chancellor's Animal Research Committee approved all the animal studies and confirmed that the experiments involving animals adhered to the guidelines set forth by the Shanghai Jiao Tong University School of Medicine (Shanghai, China).

2.2. Isolation of HPCs and Cell Culture. Four-week-old, male, wild-type C57BL/6J mice were fed a 50\% choline-deficient diet (Trophic, Nantong, China) plus $0.15 \%$ ethionine solution in drinking water (CDE diet). After 3 weeks on the diet, mice were anaesthetised by intraperitoneal Nembutal injection (1.5\% Pelltobarbitaium Natricum, Sigma-Aldrich; $0.1 \mathrm{ml} / 20 \mathrm{~g}$ body weight). HPCs were then isolated using a modified two-step perfusion protocol as previously described [13]. The cells were seeded in $90 \mathrm{~mm}$ culture dishes and cultured in complete William's E medium supplemented with $10 \%$ FBS, $2 \mathrm{mM}$ glutamine, $100 \mathrm{U} / \mathrm{ml}$ antibiotics, $20 \mathrm{ng} / \mathrm{ml}$ epidermal growth factor (EGF, Peprotech), $30 \mathrm{ng} / \mathrm{ml}$ human insulin-like growth factor II (IGF-II, GroPep), and $10 \mu \mathrm{g} / \mathrm{ml}$ insulin (Gibco). A week later, clones were selected by local trypsinization in clonal rings, and cells were resuspended in complete William's E medium. After culturing for 3 generations, purified HPCs were obtained and used for the following experiments.

For most experiments, HPCs were cultured in complete medium. In the indicated experiments, to detect the capacity of HPCs to secrete PDGF- $\beta$, FBS-free William's E medium was used. To analyse PDGFR- $\beta$ signalling, the PDGFR- $\beta$ inhibitor DMPQ (Abcam, Cambridge, UK) and ERK inhibitor FR180204 (Selleck, Houston, USA) were added to the medium.

2.3. Construction of YB-1 shRNA Lentiviruses and RNA Interference. The lentiviral vector LV-3 (GenePharma, Shanghai, China) containing a GFP reporter was used to express shRNA targeting the sequence of YB-1 (\#1: $5^{\prime}$-GA GAGCAAGGTAGACCAGTGA- $3^{\prime}$ and $\# 2: 5^{\prime}$-GTCAAAT GGTTCAATGTAAGG-3 $3^{\prime}$ ) and scramble control $\left(5^{\prime}\right.$-TTCT CCGAACGTGTCACGT-3'). Briefly, LV-3-shYB-1 plasmids were transfected into HEK 293T cells with packaging vectors. The supernatant containing infective lentiviruses was collected $72 \mathrm{~h}$ posttransfection, and the lentiviruses were concentrated by ultracentrifugation for $2 \mathrm{~h}$ at $100,000 \times \mathrm{g}$ and resuspended in PBS. HPCs were seeded in a 24 -well plate well and infected with lentiviruses in the presence of $5 \mu \mathrm{g} / \mathrm{mL}$ of polybrene. YB-1-knockdown cells were screened with $2 \mu \mathrm{g} / \mathrm{mL}$ puromycin for 15 days postinfection.

2.4. Chromatin Immunoprecipitation Assays. Preparation of chromatin and chromatin immunoprecipitation (ChIP) assays were performed using a ChIP assay kit (Upstate, NY, USA) according to the kit protocol. Chromatin equal to $2 \times 10^{6}$ cells was used for each immunoprecipitation. Chromatin immunoprecipitations were performed using HPC chromatin with normal rabbit IgG (negative control), anti-RNA polymerase II (positive control), and anti-YB-1 antibody (Abcam, ab76149). Precleared chromatin was immunoselected, processed, and subjected to PCR amplification with platinum Taq polymerase (Invitrogen, Carlsbad, CA) using sequence-specific PCR primers to amplify the region of the PDGFR- $\beta$ promoter containing the YB-1 binding site. For positive control, primers specific for the GAPDH promoter containing the RNA polymerase II binding site were applied. Detailed information regarding the primers is listed in Table 1. Reaction products were subjected to Tris/agar gel electrophoresis. Gels were stained for $5 \mathrm{~min}$ in $1 \mu \mathrm{g} /$ $\mathrm{ml}$ ethidium bromide (BioRad, Hercules, CA) and visualized using a ChemiScope 2850 imaging system (CLiNX, Shanghai, China).

For chromatin immunoprecipitation sequencing (ChIP sequencing) assays, $1 \times 10^{7}$ cells were used for each immunoprecipitation. ChIP sequencing was performed using an Illumina HiSeq2500 platform. Subsequent peak calling and motif analysis were conducted using HOMER, a software suite for ChIP-seq analysis.

2.5. Plasmid Construct, Transient Transfection, and DualLuciferase Assay. The pGL4-PDGFR- $\beta$-luciferase promoter reporter construct contained a PDGFR- $\beta$ promoter sequence 
TABLE 2: Primer sequences for RT-PCR analyses of gene expression.

\begin{tabular}{lcc}
\hline Target & Forward primer & Reverse primer \\
\hline YB-1 & CAACAGGAATGACACCAAGG & TCAACAACATCAAACTCCACAG \\
PDGF- $\beta$ & CAAGTGTGAGACAGTAGTG & ATGGGTGTGCTTAAACTTT \\
PDGFR- $\beta$ & CAAAGGTGCTGGAGATGTTG & CAGTTGTTGCTGTCCGTGTT \\
Collal & CAAGAAGACATCCCTGAAG & GCAGATACAGATCAAGCATA \\
Col4al & AGAGGAGGTGTATAGATAGC & GGAAGTCAGTCATTCAGTC \\
Laminin & CTTGGAACTGTTGGTAAGATA & ATCCTAATGAGCGGTTA \\
MMP13 & GTGTGGAGTTATGATGATGT & ACTCTCACAATGCGATTAC \\
TIMP-1 & ATCAACGAGACCACCTTA & CATATCCACAGAGGCTTTC \\
GAPDH & ACCACAGTCCATGCCATCAC & TCCACCACCCTGTTGCTGTA \\
\hline
\end{tabular}

spanning base pairs -1322 to +173 from the transcription start site ligated upstream of the luciferase gene in the pGL4 vector (Promega, Fitchburg, USA).

YB-1 knockdown or scramble control HPCs were cotransfected with the pGL4-PDGFR- $\beta$ constructs and pRL-CMV Renilla luciferase control reporter constructs using Lipofectamine 3000 reagent (Invitrogen, Carlsbad, CA) according to the manufacturer's protocol. Following $48 \mathrm{~h}$ of incubation, the cells were rinsed twice with PBS, and dual-luciferase assays were performed according to the manufacturer's protocol (Promega). Transcriptional activities of reporter constructs were normalized against those of cotransfected pRL-CMV Renilla luciferase control reporter vectors (Promega, Fitchburg, USA).

2.6. Western Blotting Analysis. Whole-cell lysates were prepared from culture cells. Medium supernatant protein was obtained by ultracentrifugation using Amicon Ultra-15 $10 \mathrm{~K}$ centrifugal filter devices (Millipore, MA, USA). Cellular or supernatant protein $(20 \mu \mathrm{g}$ per well) was loaded into $10 \%$ acrylamide gels, and then, electrophoresed proteins were transferred to PVDF membranes, and membranes were blocked for $30 \mathrm{~min}$ in TBST containing 5\% BSA. Primary antibodies were incubated for $12 \mathrm{~h}$ at $4^{\circ} \mathrm{C}$ and washed three times with TBST, followed by $2 \mathrm{~h}$ of incubation with specific IgG-HRP used at 1 in 6000 dilution in 1\% BSA-TBST. After washing, bands were detected with an ECL system.

2.7. RNA Extraction, RT-PCR, and RNA Sequencing. Total RNA was isolated using TRIzol reagent (Invitrogen). Reverse transcription was performed using PrimeScript ${ }^{\mathrm{TM}}$ RT Master Mix (Takara, Dalian, China). All primer sets for PCR are listed in Table 2. PCR was performed using SYBR ${ }^{\circledR}$ Premix Ex Taq $^{\mathrm{TM}}$ (Takara, Dalian, China) under the following conditions: 1 cycle at $95^{\circ} \mathrm{C}$ for $2 \mathrm{~min}, 35$ cycles at $95^{\circ} \mathrm{C}$ for $15 \mathrm{sec}$ and at $59^{\circ} \mathrm{C}$ for $34 \mathrm{sec}$, and $1 \mathrm{~min}$ at $72^{\circ} \mathrm{C}$. The relative mRNA expression levels were normalized against those of the GAPDH gene in the same RNA preparation.

For RNA sequencing, RNA isolation was performed using Qiagen RNEasy Mini Kit (Qiagen, Hilden, Germany) according to the protocol provided by the manufacturer, and RNA sequencing was performed using an Illumina HiSeq2500 platform.
TABle 3: Antibodies used in Western blot (WB) analysis and immunofluorescence (IF)

\begin{tabular}{|c|c|c|c|}
\hline Antibody & Dilution & Supplier & Product ID \\
\hline YB-1 & $\begin{array}{c}1: 10,000(\mathrm{WB}) \\
1: 1000(\mathrm{IF})\end{array}$ & Abcam & ab76149 \\
\hline p-YB-1 (ser102) & $1: 2000(\mathrm{WB})$ & CST & 2900 \\
\hline AFP & $1: 500$ (IF) & Abcam & ab46799 \\
\hline Albumin & $1: 500$ (IF) & Bethyl & A90-134A \\
\hline CK19 & $1: 500$ (IF) & Abnova & MAB12076 \\
\hline CD133 & $1: 100$ (IF) & eBioscience & $14-1331-80$ \\
\hline CD90/Thy1 & $1: 500$ (IF) & Novus & NB100-65543 \\
\hline$\alpha$-SMA & $1: 500$ (IF) & Abcam & ab7817 \\
\hline Collagen I & $\begin{array}{c}1: 1000(\mathrm{WB}) \\
1: 200(\mathrm{IF})\end{array}$ & Abcam & 6308 \\
\hline PDGF- $\beta$ & $1: 2000(\mathrm{WB})$ & Abcam & ab178409 \\
\hline PDGFR- $\beta$ & $1: 2000(\mathrm{WB})$ & Abcam & ab32570 \\
\hline p-Stat3 & $1: 1000(\mathrm{WB})$ & CST & 9145 \\
\hline ERK1/2 & $1: 1000(\mathrm{WB})$ & CST & 4695 \\
\hline p90 RSK (ser380) & $1: 1000(\mathrm{WB})$ & CST & 9341 \\
\hline PI3K p85 & $1: 1000(\mathrm{WB})$ & $\mathrm{R} \& \mathrm{D}$ & MAB2998 \\
\hline GAPDH & $1: 10,000(\mathrm{WB})$ & Abcam & ab8245 \\
\hline
\end{tabular}

2.8. Immunofluorescence Staining and Confocal Microscopy. The liver tissue was fixed in formalin and embedded in paraffin. Then, $4 \mu \mathrm{m}$ specimens were prepared. After antigen retrieval with Tris-EDTA (pH 9.0) for $10 \mathrm{~min}$ at $95^{\circ} \mathrm{C}$, the specimens were blocked with $5 \%$ bovine serum albumin (BSA) for $30 \mathrm{~min}$ at room temperature. The primary antibodies were applied overnight at $4^{\circ} \mathrm{C}$ in $1 \%$ BSA. Information regarding the primary antibodies is listed in Table 3 . The secondary, fluorescently labelled antibodies were applied for $1 \mathrm{~h}$ at room temperature. Sections were counterstained with 4',6-diamidino-2-phenylindole (DAPI). Fluorescence images were acquired with a Leica TCS microscope.

For cell labelling, cells were cultured on $0.2 \%$ poly-Llysine-coated glass coverslips. After fixation in $4 \%$ paraformaldehyde for $20 \mathrm{~min}$ at room temperature, cells were permeabilized in PBS containing 0.1\% Triton-100 and 5\% BSA and incubated with specific primary antibodies overnight at $4^{\circ} \mathrm{C}$. Then, the cells were stained with specific, 
fluorescently labelled secondary antibodies for $1 \mathrm{~h}$ at room temperature. After staining with DAPI and washes, confocal laser scanning microscopy and double immunofluorescence assays were performed using a Leica TCS microscope.

2.9. Gene Ontology (GO) Analysis. GO analysis was performed to analyse the main function of the DEGs according to gene ontology which is the key functional classification of NCBI. Fisher's exact test and $\chi^{2}$ test were used to classify the GO category, and the false discovery rate (FDR) was calculated to correct the $P$ value; the smaller the FDR, the smaller the error was in judging the $P$ value. The significant GO terms were defined with a $P$ value of 0.05 and an FDR $<0.05$.

2.10. Pathway Analysis. Pathway analysis was used to determine the significant pathways of the DEGs according to KEGG. Similarly, we used Fisher's exact test and $\chi^{2}$ test to select the significant pathways, and the threshold of significance was defined by $P$ value and FDR. The significant pathways were identified with a $P$ value $<0.05$ and an $\mathrm{FDR}<0.05$.

2.11. Statistical Analysis. The data represent the mean \pm SE. Statistical significance was assessed using one-way ANOVA and $t$-tests, and analysis was facilitated by the GraphPad InStat software program version 3.0.

\section{Results}

3.1. Defining the YB-1 Cistrome in HPCs. To clarify the biological functions of YB-1, we analysed the genome-wide binding sites of YB-1 in HPCs using chromatin immunoprecipitation coupled with high-throughput deep sequencing (ChIP sequencing). The resulting cistrome identified 8524 binding sites. The majority of binding sites were localized to distant intergenic and intronic regions, 3609 (62\%) and 1821 (31.5\%), respectively. The rest bind to the exon $(202,3.4 \%)$ and promoter regions (192, 3.1\%), as shown in Figure 1(a) and Supplementary Table 1 available online at https://doi. org/10.1155/2017/6193106. Gene ontology (GO) analysis of these annotated genes revealed that the most common functions for YB-1 target genes were regulation of signalling and regulation of response to stimulus. In addition, these target genes participate in cell adhesion (Figure 1(b)). Pathway analysis based on KEGG indicated that YB-1 target genes involve a variety of extremely important pathways, including metabolic pathways, the MAPK signalling pathway, cell adhesion molecules, the wnt signalling pathway, focal adhesions, and the hedgehog signalling pathway (Figure 1(c)).

3.2. Genome-Wide Expression Profiling of HPCs after YB-1 Knockdown. To further determine the effects of YB-1 in HPCs, RNA sequencing was performed to screen the differentially expressed genes (DEGs) between YB-1 knockdown HPCs and the scramble control using the following criteria: log2-fold change $>0.585$ or log2-fold change $<-0.585$, and $P$ value $<0.05$. Overall, we detected 691 DEGs between YB-1 knockdown HPCs and the scramble control, as shown in Supplementary Table 2. Among these, 395 genes were upregulated in the YB-1 knockdown HPCs and 296 genes were downregulated (Figure 2(a)). Additionally, we performed GO analysis and pathway analysis of the DEGs. Specifically, DEGs were associated with multiple GO terms, including anatomical structure morphogenesis, anatomical structure development, cell proliferation, and cell adhesion (Figure 2(b)). Pathway analysis revealed that the DEGs were involved in focal adhesions, the TGF-beta signalling pathway, ECM-receptor interaction, cell adhesion molecules, the hedgehog signalling pathway, and the wnt signalling pathway (Figure 2(c)). In addition, we found that a number of DEGs involved in ECM-receptor interaction processes were upregulated, as listed in Table 4.

3.3. YB-1 Modulates Synthesis of the Extracellular Matrix (ECM) in HPCs. Because ECM components, including collagens and laminin, are the major participants in cell adhesion, we examined if HPCs express the components involved in metabolism of the ECM. Surprisingly, we found that HPCs expressed collagen I, collagen IV, and laminin as well as the related metabolic enzymes (Figure 3(a)). Moreover, YB-1 knockdown with a lentiviral vector elevated the expression of collagen I, collagen IV, and laminin. In addition, YB-1 knockdown suppressed matrix metalloproteinase 13 (MMP13) and promoted the expression of tissue inhibitor of metalloprotease 1 (TIMP-1) at the mRNA level (Figure 3(a)). Western blot assays also demonstrated that knockdown of YB-1 was accompanied by decreased expression of the active phosphorylated YB-1 structure and overexpression of collagen I (Figure 3(b)). Immunofluorescence staining revealed that dispersed CK-19-positive HPCs produced collagen I (Figure 3(c)). These findings suggest that HPCs have the capacity to produce ECM and that YB-1 can negatively modulate this process.

3.4. YB-1 Negatively Regulates PDGFR- $\beta$ Transcriptionally. To investigate the detailed mechanism by which YB-1 regulates the expression of ECM components, ChIP sequencing and RNA sequencing were introduced to identify the potential target of YB-1. The ChIP-sequencing approach suggested that YB- 1 directly bound to the promoter region of PDGFR- $\beta$ ranging from the site 61044456 to 61044670 on chromatin $18,-640 \mathrm{bp}$ from the transcription start site, and ChIP-PCR confirmed that the PCR product is of the expected size, as shown in Figures 4(a) and 4(b). In addition, RNA sequencing showed that PDGFR- $\beta$ mRNA was elevated in the YB-1 knockdown HPCs compared to the scramble HPCs (see Supplementary Table 2). In addition, a variety of studies in the literature have reported that PDGFR- $\beta$ is upregulated in injured livers and contributes to liver cirrhosis $[14,15]$. Therefore, we chose to pursue PDGFR- $\beta$ as a candidate to reveal the molecular mechanism by which YB-1 regulates ECM homeostasis. Next, luciferase reporter assays were used to determine whether YB-1 affected transcription of the PDGFR- $\beta$ promoter. Reporter constructs containing the PDGFR $-\beta$ promoter spanning the site -1322 to +173 cloned upstream of the luciferase gene were transiently transfected into the scramble HPCs and YB-1 knockdown HPCs. The data suggested that YB-1 knockdown promoted PDGFR- $\beta$ 


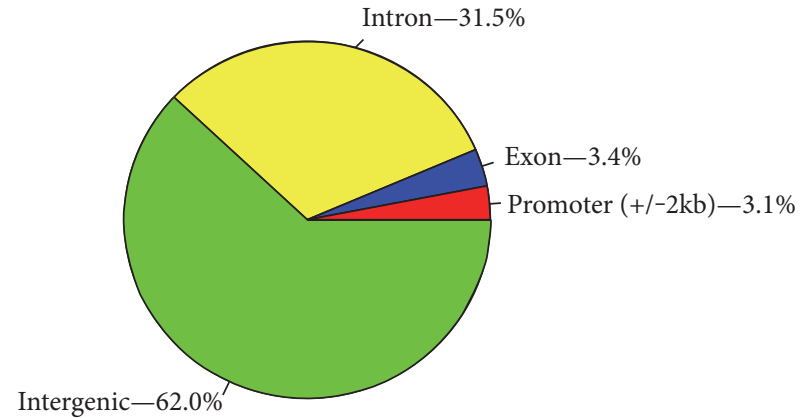

(a)

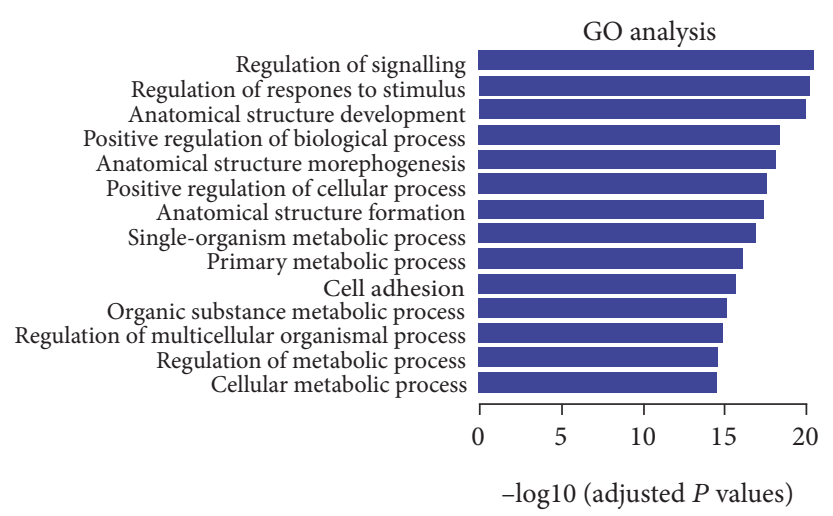

(b)

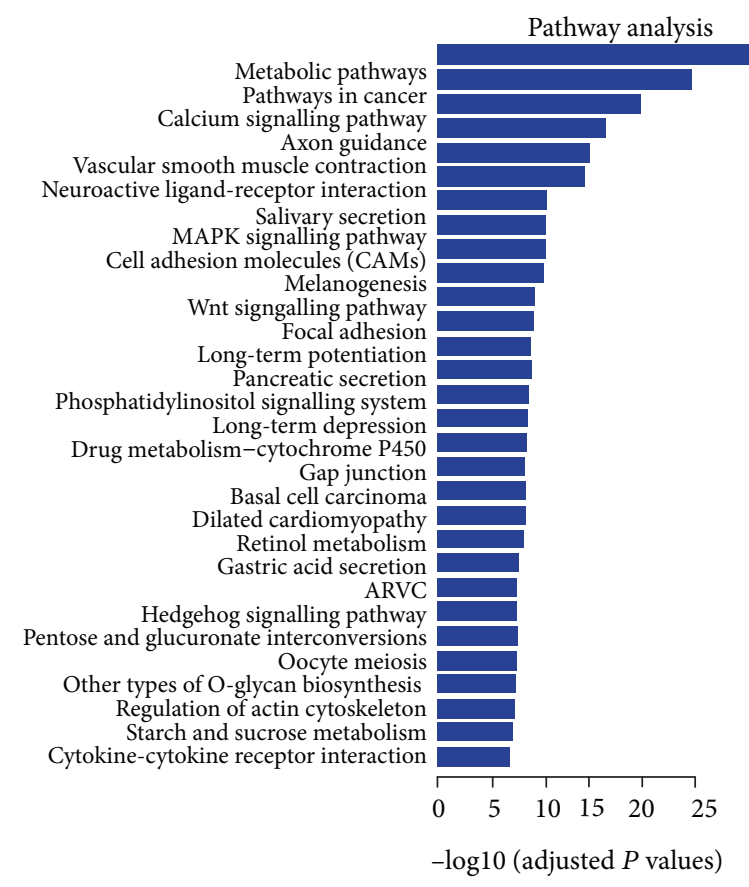

(c)

FIgure 1: The YB-1 cistrome in hepatic progenitor cells. (a) Pie chart illustrating genomic locations of YB-1 binding sites in hepatic progenitor cells. Promoter regions, $\pm 2 \mathrm{~kb}$ from the TSS. (b) Gene ontology (GO) analysis of genes annotated with YB-1 binding sites. (c) Pathway analysis of genes annotated with YB-1 binding sites.

promoter activity (Figure 4(c)). We also demonstrated that YB-1 silencing increased the expression of PDGFR- $\beta$ and its ligand PDGF- $\beta$ both in the cytoplasm and the culture medium (Figures $4(\mathrm{~d})$ and $4(\mathrm{e})$ ).

\subsection{YB-1 Affects Collagen I Expression via PDGFR- $\beta / E R K /$} p90RSK Signalling. Because YB-1 negatively regulates both collagen I and PDGFR- $\beta$, we sought to investigate whether PDGFR- $\beta$ could modulate the expression of collagen I. In this study, the PDGFR- $\beta$ inhibitor DMPQ was utilized to block the PDGFR- $\beta$ signalling pathway. Surprisingly, the effect of YB-1 knockdown on the production of collagen I was abolished by the PDGFR- $\beta$ inhibitor in a dosedependent manner (Figure 5(a)). Meanwhile, we detected the key components of PDGFR- $\beta$-related signalling pathways that may participate in the expression of collagen I in HPCs.
Western blotting assays showed that phosphorylated ERK1/2 but not phosphorylated PI3K or Stat3 mediated PDGFR- $\beta$ induced collagen I production (Figure 5(b)). Further studies demonstrated that phosphorylation of p90 RSK at Ser380 synchronized with the amount of phosphorylated ERK1/2 and collagen I (Figure 5(c)).

\section{Discussion}

YB-1 has been reported to be elevated in HCC tissues and in regenerating liver after injury $[9,10]$. In this study, ChIP sequencing and RNA sequencing provided clues that YB-1 participated in the biological adhesion process and ECMreceptor interaction in HPCs. Therefore, we detected the ligand components, including collagen and laminin, involved in the biological process. Surprisingly, we found that HPCs 


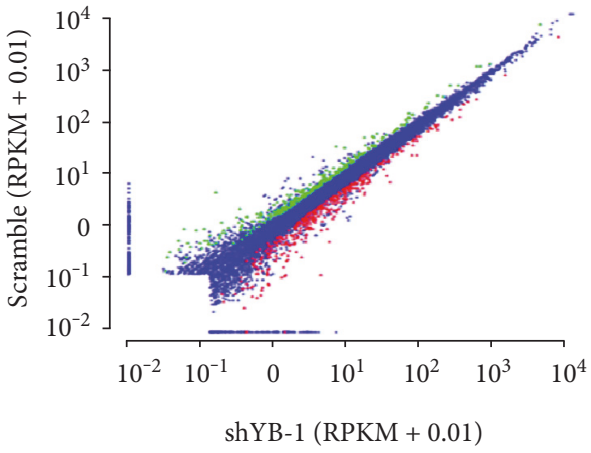

- Upregulated: 395

- Not changed: 15,748

- Downregulated: 296

(a)

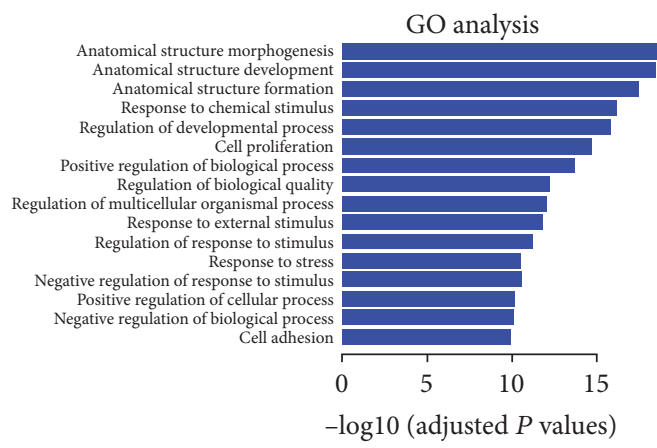

(b)

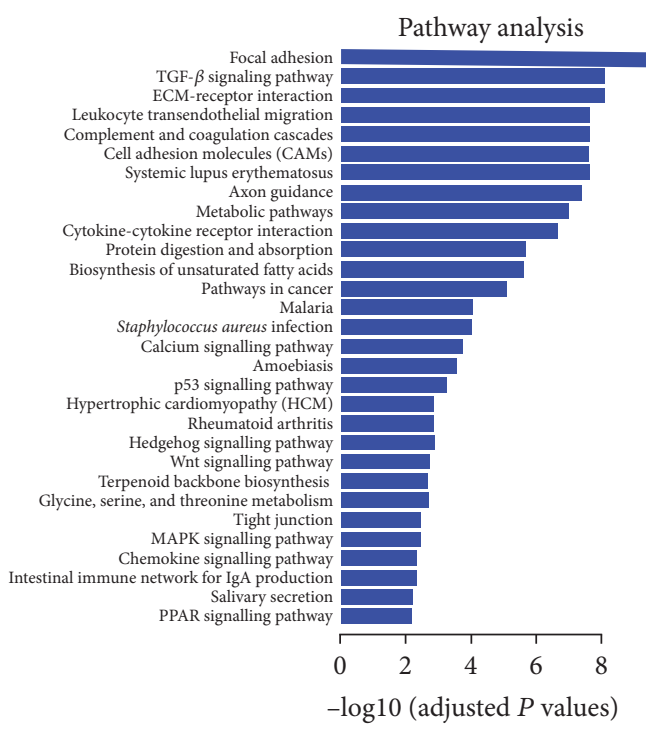

(c)

FIGURE 2: Differentially expressed genes (DEGs) of HPCs with YB-1 knockdown. (a) Overview of DEGs of HPCs with YB-1 knockdown. Log2-fold change $>0.585$ or Log2-fold change $<-0.585$, and $P$ value $<0.05$. (b) Gene ontology (GO) analysis of DEGs. (c) Pathways enrichment analysis of DEGs based on KEGG.

TABLE 4: Transcripts upregulated in ECM-receptor interaction.

\begin{tabular}{lcccc}
\hline Gene name & Gene & Fold change & YB-1 KD-RPKM & Scramble-RPKM \\
\hline Collagen, type III, alpha 1 & Col3a1 & 4.58 & 1.89125 & 0.412824 \\
Collagen, type V, alpha 2 & Col5a2 & 3.40 & 4.68447 & 1.37872 \\
Thrombospondin 2 & Thbs2 & 2.15 & 0.414252 & 0.193934 \\
Reelin & Reln & 1.89 & 0.916106 & 0.48511 \\
Thrombospondin 1 & Thbs1 & 1.79 & 93.503 & 52.3547 \\
Collagen, type I, alpha 2 & Colla2 & 1.78 & 5.134 & 2.87674 \\
Collagen, type IV, alpha 2 & Col4a2 & 1.69 & 119.857 & 70.8253 \\
Collagen, type IV, alpha 1 & Col4a1 & 1.63 & 202.428 & 123.942 \\
Integrin beta 6 & Itgb6 & 1.61 & 6.48106 & 4.03229 \\
\hline
\end{tabular}

produced collagen and laminin. Moreover, silencing YB-1 promoted the expression of collagen I and laminin. ChIP sequencing and RNA sequencing identified PDGFR- $\beta$ as a candidate target of YB-1, and the result was confirmed by ChIP-PCR, which indicated that YB-1 was able to directly bind to the PDGFR- $\beta$ promoter. In addition, 


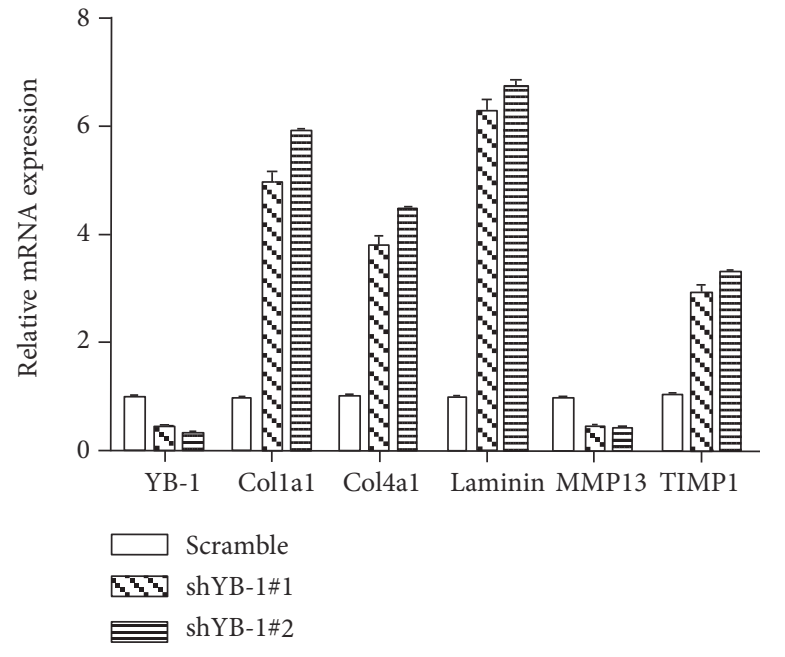

(a)

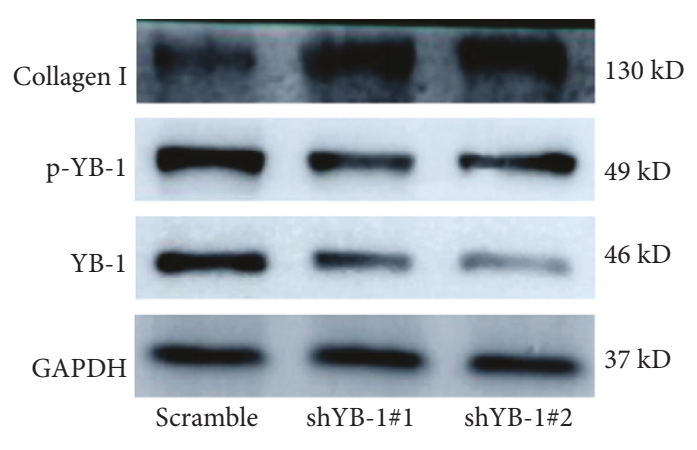

(b)

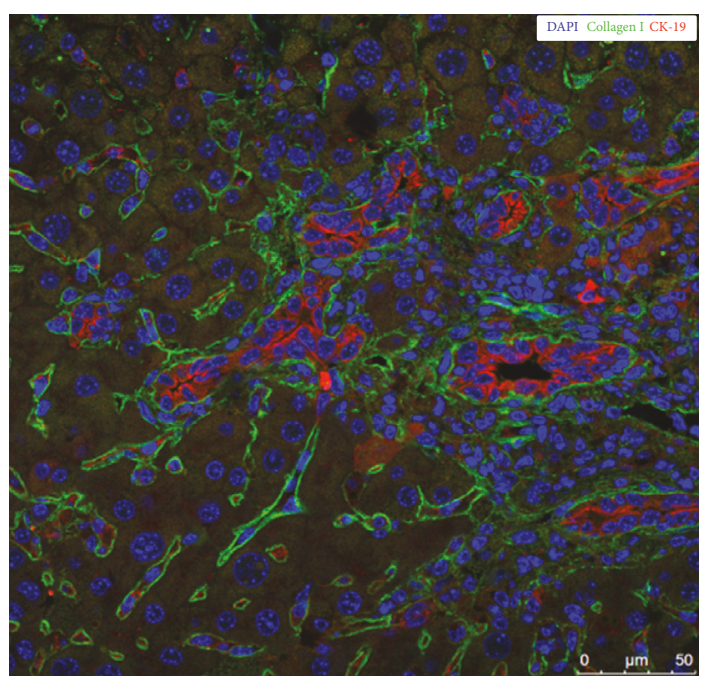

(c)

FIGURE 3: YB-1 regulates the synthesis of ECM proteins. (a) YB-1 knockdown promoted the mRNA expression of Col1a1, Col4a1, laminin, and TIMP-1, but suppressed the expression of MMP13. (b) Western blot assays provided evidence that YB-1 knockdown enhanced collagen I expression in HPCs. (c) CDE-injured mouse liver sections were stained with CK-19 and collagen I, and the results showed that collagen I can be expressed by a number of single dispersed CK-19-positive cells, which represented a subpopulation of HPCs.

luciferase reporter assays demonstrated that YB-1 negatively modulated $P D G F R-\beta$ promoter activity. Because PDGFR- $\beta$ plays an extremely important role in liver fibrogenesis, we investigated its role in collagen expression in HPCs. We found that PDGF- $\beta$ and PDGFR- $\beta$ showed a similar trend with collagen I, and a PDGFR- $\beta$ inhibitor and ERK1/2 inhibitor blunted collagen I expression after YB-1 knockdown.

Liver fibrosis is a wound-healing response accompanied by excessive deposition of extracellular matrix, which develops to cirrhosis if the damage persists. During the past decades, HSCs have been regarded as the major offenders contributing to liver fibrosis $[12,16]$. In addition, other fibrogenic cells are reported to be involved, such as portal fibroblasts [17], fibrocytes [18], bone-marrow-derived cells [19], and cells derived from epithelial mesenchymal transition [20]. Moreover, HPCs express ECM-related genes [21].
Van Hul and colleagues reported that HPCs are embedded in ECM at all times, demonstrating that HPCs have the potential to synthesize ECM and cause hepatic fibrosis [22]. In this article, we provide more persuasive evidence to support the notion that HPCs also yield ECM, and this evidence is beneficial for completely uncover the mechanism of fibrogenesis. Due to the ability to differentiate into mature hepatocytes and cholangiocytes [23], HPCs are regarded as a reservoir to compensate for damaged hepatocytes or cholangiocytes, especially under perpetual injury conditions $[24,25]$. When mice were fed a $50 \%$ choline-deficient, $0.15 \%$ ethionine-supplemented diet for 2 weeks, abundant HPCs, representing approximately $28 \%$ of the total liver cells, appeared around the periportal region of the liver. Based on these data, we proposed a new model in which HPCs contribute to liver fibrogenesis because of their vigorous proliferation rate and capacity to synthesize ECM. 


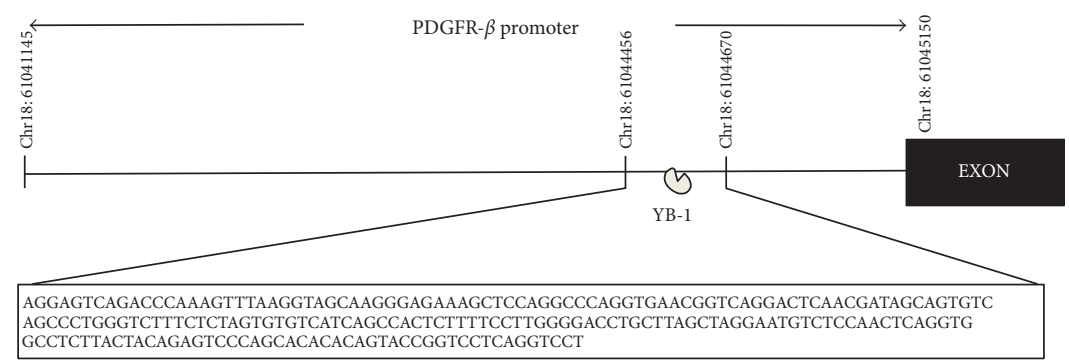

(a)
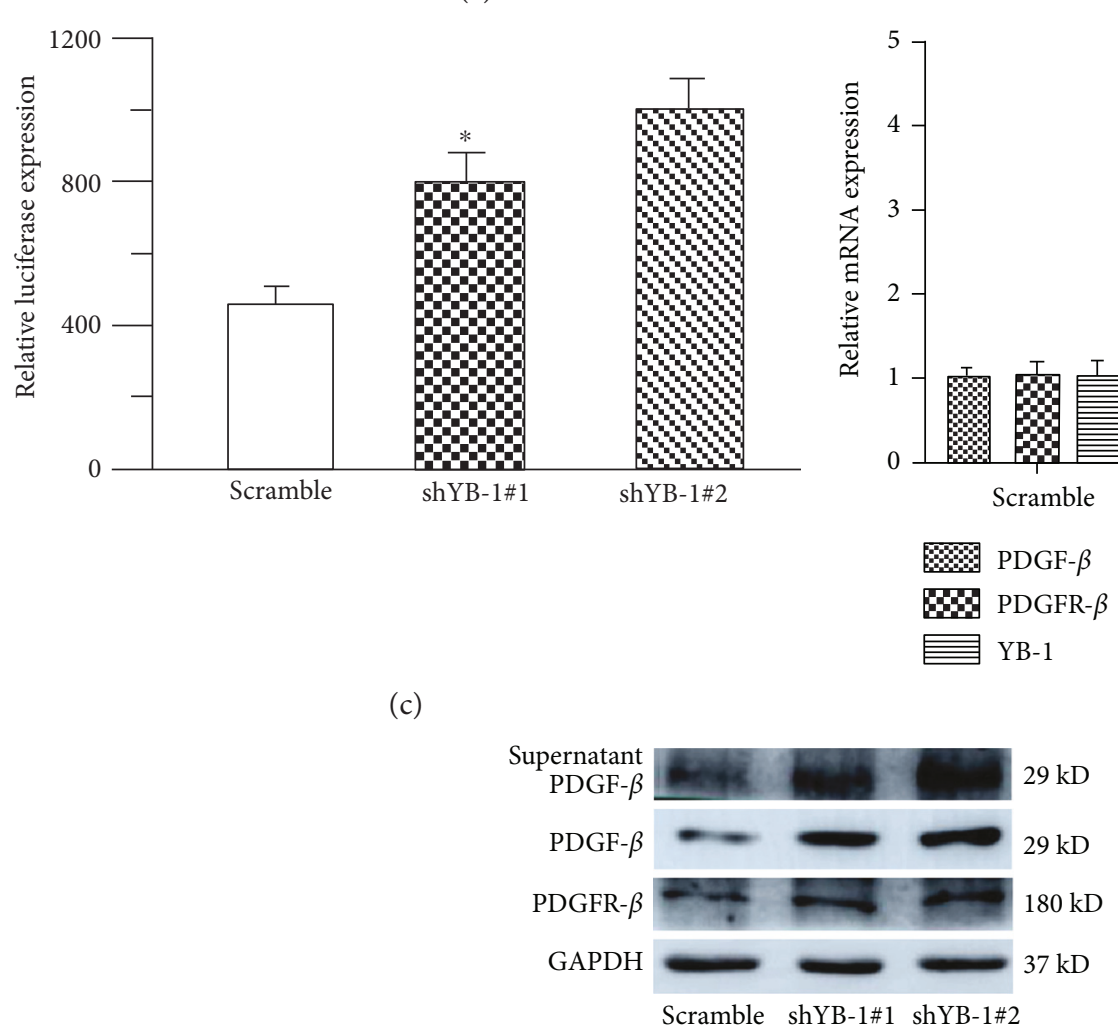

(e)

(c)

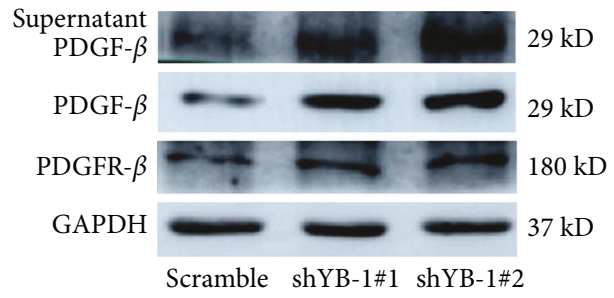

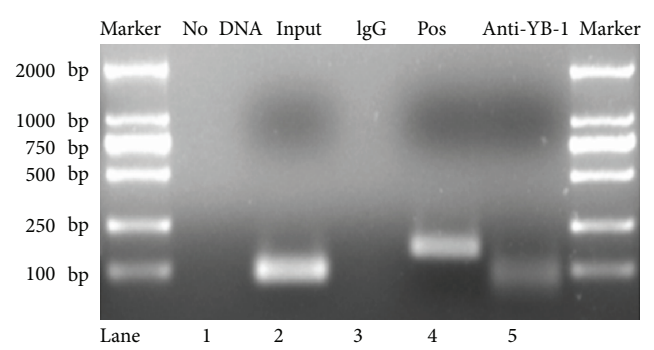

(b)

(d)

FIGURE 4: YB-1 directly binds to the PDGFR- $\beta$ promoter and negatively regulates its expression. (a) ChIP-sequencing assays detected that YB-1 directly bound to a region of the PDGFR- $\beta$ promoter spanning from the site 61044456 to 61044670 on chromatin 18. (b) ChIP-PCR confirmed that PDGFR- $\beta$ is one of the YB-1 target genes. Chromatin immunoprecipitations were performed using HPC chromatin with normal rabbit IgG, anti-RNA polymerase II, and anti-YB-1 antibodies. Purified DNA was then analysed with PCR using primers specific for YB-1 binding sites on the PDGFR- $\beta$ promoter or primers for the GAPDH promoter as the positive control. The size of the PCR product for the PDGFR- $\beta$ promoter and the GAPDH promoter was 82 and $166 \mathrm{bp}$, respectively. The PCR product was observed in the input, positive, and anti-YB-1 ChIPs (lanes 2, 4, and 5) but not in the no DNA and IgG ChIPs (lanes 1 and 3). (c) YB-1 negatively modulates PDGFR- $\beta$ promoter activity in HPCs. HPCs were transfected with a scramble lentivirus or YB-1 knockdown lentivirus; PDGFR- $\beta$ promoter-luciferase constructs and Renilla luciferase constructs were cotransfected into HPCs. Cells were harvested $48 \mathrm{~h}$ after the second transfection, and luciferase activity was measured. The results are an average of three independent transfections. (d) YB-1 knockdown enhanced PDGFR- $\beta$ mRNA expression. In addition, PDGF- $\beta$ expression was elevated in HPCs with YB-1 silenced. (e) Western blotting revealed that YB-1 knockdown negatively regulated PDGFR- $\beta$ and PDGF- $\beta$ expression. Compared with the culture supernatant from HPCs transfected with the scrambled lentivirus, the YB-1 knockdown groups showed higher levels of PDGF- $\beta$ protein. ${ }^{*} P<0.01$, compared with the scramble control.

YB-1 was originally identified as a transcription factor that regulates the expression of numerous genes, including p53, MDR1, EGFR, cyclin A, and cyclin D1. The biological activities in which YB-1 participates range from cell proliferation, differentiation and malignant cell transformation to cell adhesion. Merterns et al. reported that YB-1 directly bound to the promoter of the collagen $\alpha 1$. (I) gene and suppressed its promoter activity in human fibroblasts. Higashi and coworkers reported that YB-1 counter-repressed TGF$\beta$-stimulated collagen $\alpha 2$ (I) transcription by interfering with Smad3 bound upstream of p300 in human dermal fibroblasts [26]. In addition, YB-1 was described to bind to the Smad7 


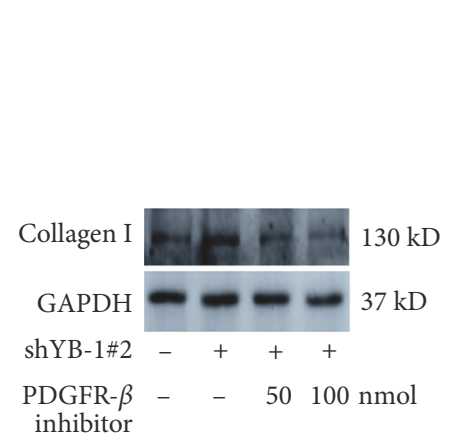

(a)

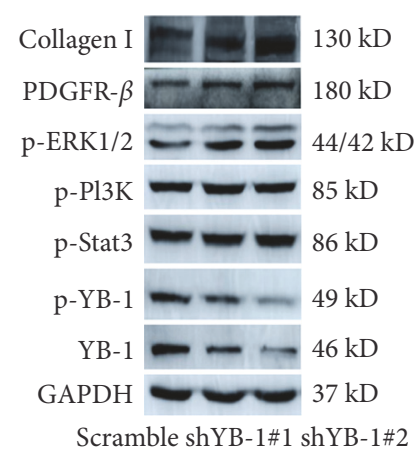

(b)

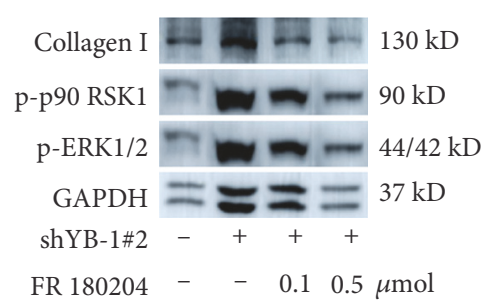

(c)

FIGURE 5: YB-1 regulates collagen I expression via PDGFR- $\beta /$ ERK/p90RSK signalling. (a) YB-1 silencing enhanced collagen I expression in HPCs, but the PDGFR- $\beta$ inhibitor DMPQ blunted the regulatory effect of YB-1 silencing on collagen I expression. (b) Seven days after YB-1 knockdown, HPCs were harvested to analyse the signalling pathway involved in YB-1-regulated collagen I expression. Following YB-1 silencing, phosphorylated YB-1 also decreased, while PDGFR- $\beta$ and phosphorylated ERK1/2 were elevated. The level of phosphorylated Stat3 and PI3K showed no alteration. (c) The ERK1/2 inhibitor FR180204 repressed phosphorylation of p90RSK1 and ERK1/2 and abolished YB-1-regulated collagen I expression.

promoter and induce the expression of Smad7 in rat HSCs, further inhibiting collagen expression [11]. These data suggest that YB-1 plays an inhibitory role in collagen expression, indicating that it may contribute to the fibrogenesis process. Thus, we sought to explore the effect of YB-1 on hepatic fibrogenesis in which activated HSCs play a predominant role. Herein, we identified that YB-1 knockdown with shYB-1 lentivirus vector promoted collagen I expression in HPCs through upregulation of PDGFR- $\beta$, which is different from the above mechanisms in which YB-1 modulates collagen I expression. In this study, collagen I was found to not be a target gene of YB-1 using ChIP sequencing (see Supplementary Table 1), which excludes the possibility that YB-1 binds to collagen I and regulates its expression.

For the first time, we identified PDGFR- $\beta$ as a target gene of YB-1. To our knowledge, PDGF receptor signal transduction plays important roles in the embryo development, angiogenesis, and fibrotic diseases as well as in tumour development and migration $[16,27]$. Elevated PDGF expression in cells expressing PDGFR has been demonstrated following both acute and chronic liver damage in experimental and human diseases. During liver fibrogenesis, PDGF- $\beta$ has been recognized as the most potent mitogen for HSCs. In addition, PDGF- $\beta$ is identified as a profibrogenic stimulus of HSCs using a transgenic mouse model [14]. However, our data demonstrate that the PDGF signalling network is involved in collagen synthesis in HPCs. To date, few articles have reported that PDGF signalling pathways are involved in the biological functions of HPCs. Only Lau et al. reported that the PDGFR$\beta$ inhibitor AG1296 suppressed the viability of a tumourigenic hepatic progenitor cell line (PIL2), which arises from p53 knockout in HPCs. In the present study, we show that HPCs express both PDGF- $\beta$ and PDGFR- $\beta$, indicating that there may be an autocrine regulatory loop that modulates collagen synthesis in HPCs [28]. In addition, a serum-free culture system confirmed that HPCs secreted PDGF- $\beta$, and a PDGFR- $\beta$ inhibitor inhibited the collagen production by HPCs. Further study demonstrated that ERK/p90RSK signalling mediates PDGFR- $\beta$-induced collagen expression in HPCs.
In conclusion, this study presents a novel mechanism in which YB-1 negatively modulates the expression of ECM in HPCs by negatively regulating the transcription of PDGFR- $\beta$. Disruption of the PDGFR- $\beta /$ ERK/p90RSK signalling pathway represses the collagen expression in HPCs. The findings will help to uncover the mechanism of liver fibrogenesis, and interference with the regulatory axis may provide a new approach for treatment of liver fibrosis and cirrhosis.
Abbreviations
ChIP: Chromatin immunoprecipitation
ECM: Extracellular matrix
EGF: Epidermal growth factor receptor
ERK: $\quad$ Extracellular signal-regulated kinase
HPCs: Hepatic progenitor cells
HSCs: Hepatic stellate cells
PDGF: Platelet-derived growth factor
PDGFR: Platelet-derived growth factor receptor
RSK: Ribosomal S6 kinase
RT-PCR: Reverse transcriptase polymerase chain reaction
TGF: Transforming growth factor
YB-1: $\quad$ Y-box protein-1.

\section{Additional Points}

Highlights. (1) Hepatic progenitor cells synthesize extracellular matrix. (2) YB-1 directly binds to the PDGFR- $\beta$ promoter and negatively regulates PDGFR- $\beta$ transcription. (3) YB-1 knockdown enhances collagen I expression via PDGFR- $\beta$ / ERK/p90RSK signalling.

\section{Conflicts of Interest}

There are no conflicts of interest. 


\section{Authors' Contributions}

Fei Li and Zhenzeng Ma contributed equally to this study.

\section{Acknowledgments}

This study was supported by the National Natural Science Foundation of China (nos. 81270518 and 81470858) and the Program for Shanghai Outstanding Academic Leader (no. 2015056).

\section{References}

[1] D. K. Dedier, J. Schiffenbuer, S. L. Woulfe, M. Zacheis, and B. D. Schwartz, "Charaterization of the cDNA encoding a protein binding to the major histocompatibility complex class II Y box," PNAS, vol. 85, no. 19, pp. 7322-7326, 1998.

[2] T. Okamoto, H. Izumi, T. Imamura et al., "Direct interaction of p53 with the Y-box binding protein, YB-1: a mechanism for regulation of human gene expression," Oncogene, vol. 19, no. 54, pp. 6194-6202, 2000.

[3] A. Lasham, S. Moloney, T. Hale et al., "The Y-box-binding protein, YB1, is a potential negative regulator of the $\mathrm{p} 53$ tumor suppressor," The Journal of Biological Chemistry, vol. 278, no. 37, pp. 35516-35523, 2003.

[4] M. Harada, Y. Kotake, T. Ohhata et al., "YB-1 promotes transcription of cyclin D1 in human non-small-cell lung cancers," Genes to Cells, vol. 19, no. 6, pp. 504-516, 2014.

[5] A. Astanehe, M. R. Finkbeiner, M. Krzywinski et al., "MKNK1 is a YB-1 target gene responsible for imparting trastuzumab resistance and can be blocked by RSK inhibition," Oncogene, vol. 31, no. 41, pp. 4434-4446, 2012.

[6] A. L. Stratford, G. Habibi, A. Astanehe et al., "Epidermal growth factor receptor (EGFR) is transcriptionally induced by the Y-box binding protein-1 (YB-1) and can be inhibited with Iressa in basal-like breast cancer, providing a potential target for therapy," Breast Cancer Research, vol. 9, no. 5, p. R61, 2007.

[7] J. T. Norman, G. E. Lindahl, K. Shakib, A. En-Nia, E. Yilmaz, and P. R. Mertens, "The Y-box binding protein YB-1 suppresses collagen alpha 1 (I) gene transcription via an evolutionarily conserved regulatory element in the proximal promoter," The Journal of Biological Chemistry, vol. 276, no. 32, pp. 29880-29890, 2001.

[8] S. Harendza, D. H. Lovett, and R. A. K. Stahl, "The hematopoietic transcription factor PU.1 represses gelatinase A transcription in glomerular mesangial cells," The Journal of Biological Chemistry, vol. 275, no. 26, pp. 19552-19559, 2000.

[9] C. E. Grant and R. G. Deeley, "Cloning and characterization of chicken YB-1: regulation of expression in the liver," Molecular and Cellular Biology, vol. 13, no. 7, pp. 41864196, 1993.

[10] V. P. Gunasekaran and M. Ganeshan, "Inverse correlation of ribosomal protein S27A and multifunctional protein YB-1 in hepatocellular carcinoma," Clinical Biochemistry, vol. 47, no. 13-14, pp. 1262-1264, 2014.

[11] S. Dooley, H. M. Said, A. M. Gressner, J. Floege, A. En-Nia, and P. R. Mertens, "Y-box protein-1 is the crucial mediator of antifibrotic interferon-gamma effects," The Journal of Biological Chemistry, vol. 281, no. 3, pp. 1784-1795, 2006.
[12] Y. Inagaki, M. Kushida, K. Higashi et al., "Cell type-specific intervention of transforming growth factor $\beta /$ Smad signaling suppresses collagen gene expression and hepatic fibrosis in mice," Gastroenterology, vol. 129, no. 1, pp. 259-268, 2005.

[13] B. Akhurst, E. J. Croager, C. A. Farley-Roche et al., "A modified choline-deficient, ethionine-supplemented diet protocol effectively induces oval cells in mouse liver," Hepatology, vol. 34, no. 3, pp. 519-522, 2001.

[14] P. Czochra, B. Klopcic, E. Meyer et al., "Liver fibrosis induced by hepatic overexpression of PDGF-B in transgenic mice," Journal of Hepatology, vol. 45, no. 3, pp. 419-428, 2006.

[15] E. Borkham-Kamphorst and R. Weiskirchen, "The PDGF system and its antagonists in liver fibrosis," Cytokine o Growth Factor Reviews, vol. 28, pp. 53-61, 2016.

[16] P. Kocabayoglu, A. Lade, Y. A. Lee et al., "Beta-PDGF receptor expressed by hepatic stellate cells regulates fibrosis in murine liver injury, but not carcinogenesis," Journal of Hepatology, vol. 63, no. 1, pp. 141-147, 2015.

[17] M. N. Jhandier, E. A. Kruglov, E. G. Lavoie, J. Sévigny, and J. A. Dranoff, "Portal fibroblasts regulate the proliferation of bile duct epithelia via expression of NTPDase2," The Journal of Biological Chemistry, vol. 280, no. 24, pp. 2298622992, 2005.

[18] T. Kisseleva, H. Uchinami, N. Feirt et al., "Bone marrowderived fibrocytes participate in pathogenesis of liver fibrosis," Journal of Hepatology, vol. 45, no. 3, pp. 429-438, 2006.

[19] S. J. Forbes, F. P. Russo, V. Rey et al., "A significant proportion of myofibroblasts are of bone marrow origin in human liver fibrosis," Gastroenterology, vol. 126, no. 4, pp. 955-963, 2004.

[20] M. Beaussier, D. Wendum, E. Schiffer et al., "Prominent contribution of portal mesenchymal cells to liver fibrosis in ischemic and obstructive cholestatic injuries," Laboratory Investigation, vol. 87, no. 3, pp. 292-303, 2007.

[21] P. Wang, M. Cong, T. Liu et al., "The characteristics variation of hepatic progenitors after TGF-betal-induced transition and EGF-induced reversion," Stem Cells International, vol. 2016, Article ID 6304385, 10 pages, 2016.

[22] N. K. M. Van Hul, J. Abarca-Quinones, C. Sempoux, Y. Horsmans, and I. A. Leclercq, "Relation between liver progenitor cell expansion and extracellular matrix deposition in a CDE-induced murine model of chronic liver injury," Hepatology, vol. 49, no. 5, pp. 1625-1635, 2009.

[23] C. A. Lazaro, J. A. Rhim, Y. Yamada, and N. Fausto, "Generation of hepatocytes from oval cell precursors in culture," Cancer Research, vol. 58, no. 23, pp. 5514-5522, 1998.

[24] S. Shin, G. Walton, R. Aoki et al., "Foxl1-Cre-marked adult hepatic progenitors have clonogenic and bilineage differentiation potential," Genes \& Development, vol. 25, no. 11, pp. 1185-1192, 2011.

[25] R. Espanol-Suner, R. Carpentier, N. Van Hul et al., "Liver progenitor cells yield functional hepatocytes in response to chronic liver injury in mice," Gastroenterology, vol. 143, no. 6, pp. 1564.e7-1575.e7, 2012.

[26] K. Higashi, Y. Inagaki, K. Fujimori, A. Nakao, H. Kaneko, and I. Nakatsuka, "Interferon-gamma interferes with transforming growth factor-beta signaling through direct interaction of YB-1 with Smad3," The Journal of Biological Chemistry, vol. 278, no. 44, pp. 43470-43479, 2003. 
[27] J. B. Demoulin and A. Essaghir, "PDGF receptor signaling networks in normal and cancer cells," Cytokine \& Growth Factor Reviews, vol. 25, no. 3, pp. 273-283, 2014.

[28] C. K. Lau, Z. F. Yang, D. W. Ho et al., "An Akt/hypoxiainducible factor-1alpha/platelet-derived growth factor-BB autocrine loop mediates hypoxia-induced chemoresistance in liver cancer cells and tumorigenic hepatic progenitor cells," Clinical Cancer Research, vol. 15, no. 10, pp. 3462-3471, 2009. 

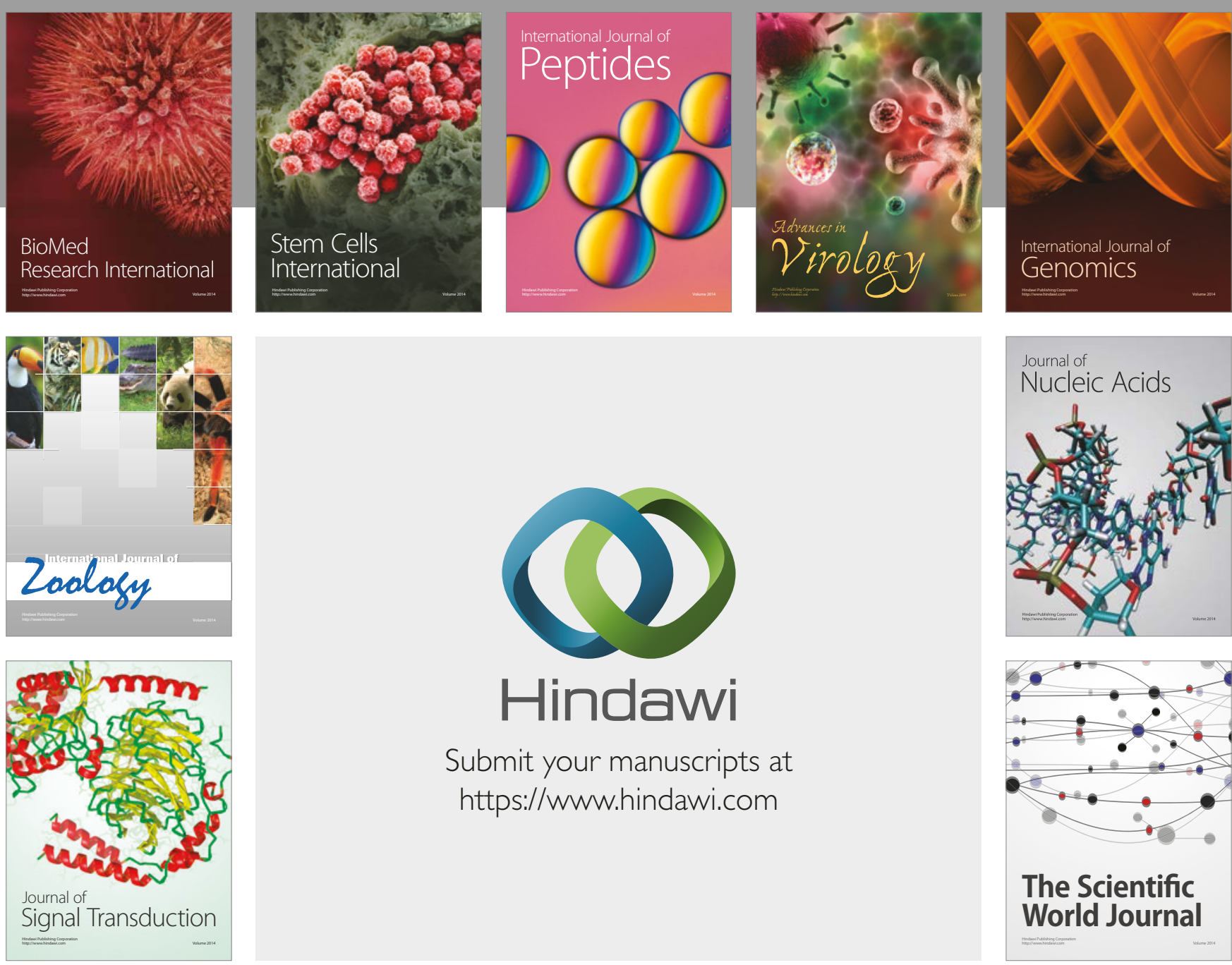

Submit your manuscripts at

https://www.hindawi.com
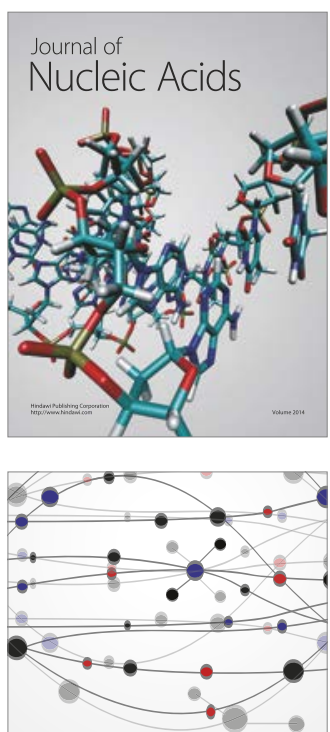

The Scientific World Journal

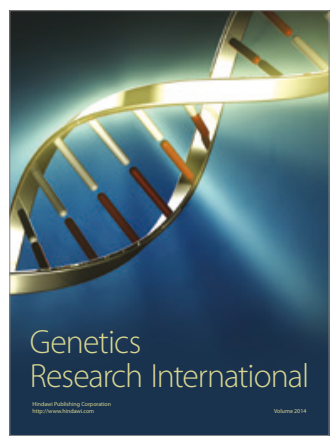

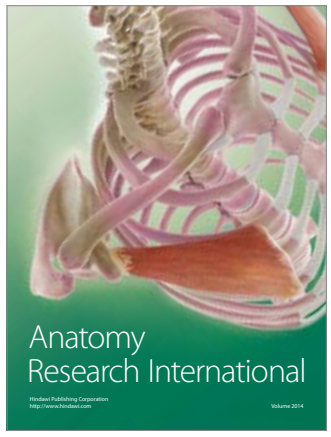

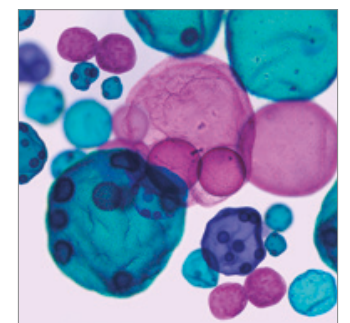

International Journal of Microbiology
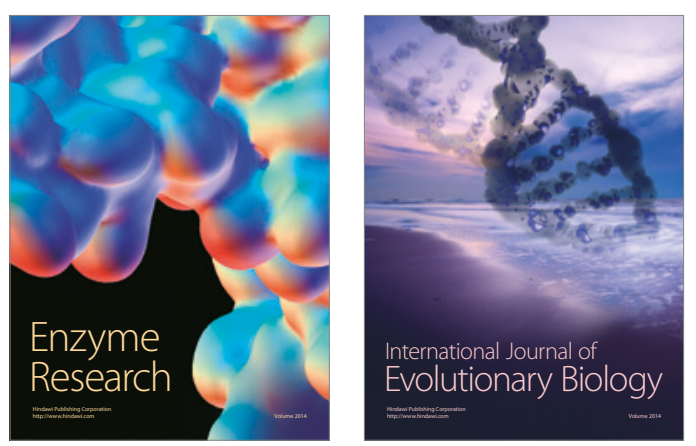
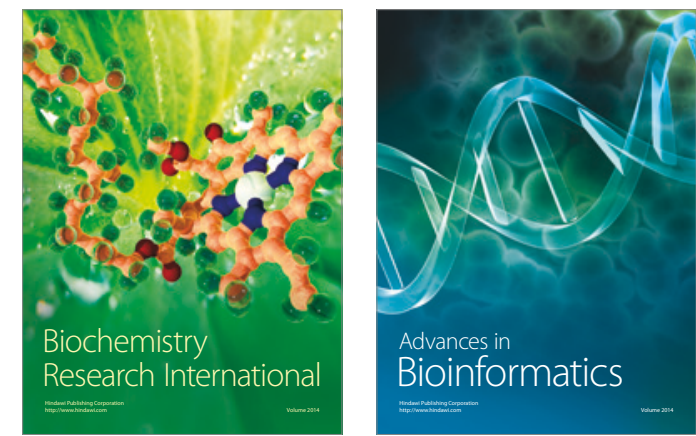

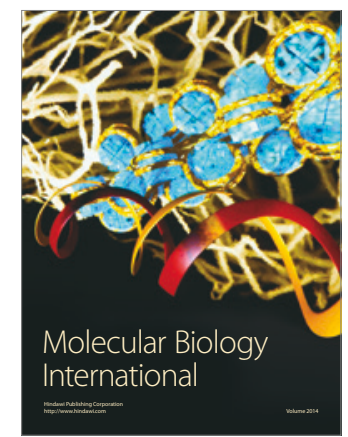

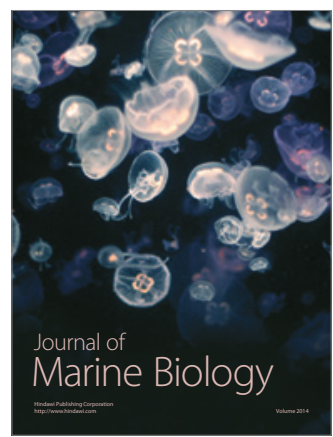

